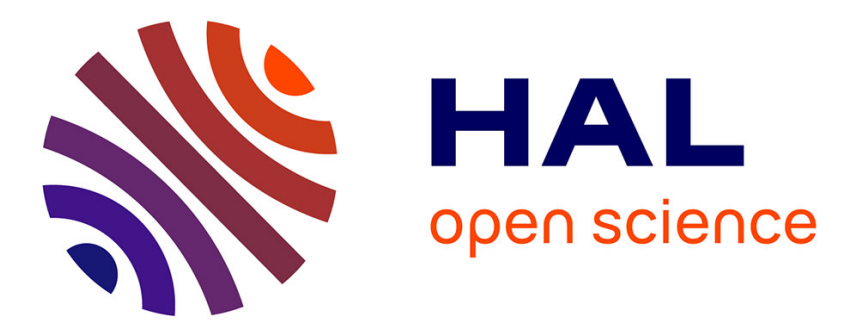

\title{
TWO COMPUTATIONS CONCERNING THE ISOVECTORS OF THE BACKWARD HEAT EQUATION WITH QUADRATIC POTENTIAL
}

\author{
Mohamad Houda, Paul Lescot
}

\section{- To cite this version:}

Mohamad Houda, Paul Lescot. TWO COMPUTATIONS CONCERNING THE ISOVECTORS OF THE BACKWARD HEAT EQUATION WITH QUADRATIC POTENTIAL. Methods and Applications of Analysis, In press. hal-02399987

\section{HAL Id: hal-02399987 \\ https://hal.science/hal-02399987}

Submitted on 9 Dec 2019

HAL is a multi-disciplinary open access archive for the deposit and dissemination of scientific research documents, whether they are published or not. The documents may come from teaching and research institutions in France or abroad, or from public or private research centers.
L'archive ouverte pluridisciplinaire $\mathbf{H A L}$, est destinée au dépôt et à la diffusion de documents scientifiques de niveau recherche, publiés ou non, émanant des établissements d'enseignement et de recherche français ou étrangers, des laboratoires publics ou privés. 


\title{
TWO COMPUTATIONS CONCERNING THE ISOVECTORS OF THE BACKWARD HEAT EQUATION WITH QUADRATIC POTENTIAL
}

\author{
MOHAMAD HOUDA \& PAUL LESCOT
}

\begin{abstract}
We determine the isovectors of the backward heat equation with quadratic potential term in the space variable. This generalize the calculations of Lescot-Zambrini (cf.[4]). These results first appeared in the first author's PhD thesis (Rouen, 2013).
\end{abstract}

\section{BACKWARD EqUATiON}

The fundamental equation of Euclidean Quantum Mechanics of Zambrini is the backward heat equation with potential $V(t, q)$ :

$$
\left\{\begin{aligned}
\theta^{2} \frac{\partial \eta_{u}^{V}}{\partial t} & =-\frac{\theta^{4}}{2} \frac{\partial^{2} \eta_{u}^{V}}{\partial q^{2}}+V(t, q) \eta_{u}^{V} \quad\left(C_{1}^{(V)}\right) \\
\eta_{u}^{V}(0, q) & =u(q)
\end{aligned}\right.
$$

where $t$ represents the time variable, $q$ the space variable, and $\theta$ is a real parameter, strictly positive (in physics, $\theta=\sqrt{\hbar}$ ).

This equation is not well-posed in general, but existence and uniqueness of a solution are insured whenever the initial condition $u$ belongs to the set of analytic vectors for the operator appearing on the right-hand side of the equation (see e.g. [1], Lemma 4, p. 429).

We shall denote by $\eta_{u}(t, q)=\eta_{u}^{0}(t, q)$ the solution of the backward heat equation with null potential :

$$
\left\{\begin{aligned}
\theta^{2} \frac{\partial \eta_{u}}{\partial t} & =-\frac{\theta^{4}}{2} \frac{\partial^{2} \eta_{u}}{\partial q^{2}} \quad\left(C_{1}^{(0)}\right) \\
\eta_{u}(0, q) & =\eta_{u}^{0}(0, q)=u(q)
\end{aligned}\right.
$$

The authors wish to thank the referees for constructive comments.

\subsection{Generalization of results due to Lescot-Zambrini (cf.[4]).}

Theorem 1.1. Let $a(t), b(t)$ and $c(t)$ be continuous functions and $V(t, q)=a(t) q^{2}+$ $b(t) q+c(t)$.

For all initial conditions $u$, the solution $\eta_{u}^{V}(t, q)$ of:

$$
\theta^{2} \frac{\partial \eta_{u}^{V}}{\partial t}=-\frac{\theta^{4}}{2} \frac{\partial^{2} \eta_{u}^{V}}{\partial q^{2}}+V(t, q) \eta_{u}^{V} \quad\left(C_{1}^{(V)}\right)
$$

such that $\eta_{u}^{V}(0, q)=u(q)$ is given by:

$$
\eta_{u}^{V}(t, q)=\varphi_{1}(t, q) \eta_{u}\left(\varphi_{2}(t, q), \varphi_{3}(t, q)\right),
$$

Date: 01 November 2019 
where: $\eta_{u}(t, q)=\eta_{u}^{0}(t, q)$ and $\varphi_{1}(t, q), \varphi_{2}(t, q), \varphi_{3}(t, q)$ only depend on $a(t), b(t)$ and $c(t)$ (via formulas (1.7), (1.8), (1.9), (1.11), (1.12), (1.13) and (1.14)).

Proof. We shall make formula 1.1 explicit with the following initial conditions:

$$
\left\{\begin{array}{l}
\varphi_{1}(0, q)=1 \\
\varphi_{2}(0, q)=0 \\
\varphi_{3}(0, q)=q
\end{array}\right.
$$

In this case :

$$
\eta_{u}^{V}(0, q)=\eta_{u}(0, q)=u(q) .
$$

We now differentiate formula 1.1 with respect to $t$ and $q$;

$\eta_{u}$ and its derivatives will be taken in $\left(\varphi_{2}(t, q), \varphi_{3}(t, q)\right)$.

$$
\begin{gathered}
\frac{\partial \eta_{u}^{V}}{\partial t}=\frac{\partial \varphi_{1}}{\partial t} \eta_{u}+\varphi_{1}\left(\frac{\partial \eta_{u}}{\partial t} \frac{\partial \varphi_{2}}{\partial t}+\frac{\partial \eta_{u}}{\partial q} \frac{\partial \varphi_{3}}{\partial t}\right) \\
\frac{\partial \eta_{u}^{V}}{\partial q}=\frac{\partial \varphi_{1}}{\partial q} \eta_{u}+\varphi_{1}\left(\frac{\partial \eta_{u}}{\partial t} \frac{\partial \varphi_{2}}{\partial q}+\frac{\partial \eta_{u}}{\partial q} \frac{\partial \varphi_{3}}{\partial q}\right), \\
\frac{\partial^{2} \eta_{u}^{V}}{\partial q^{2}}=\frac{\partial^{2} \varphi_{1}}{\partial q^{2}} \eta_{u}+2 \frac{\partial \varphi_{1}}{\partial q}\left(\frac{\partial \eta_{u}}{\partial t} \frac{\partial \varphi_{2}}{\partial q}+\frac{\partial \eta_{u}}{\partial q} \frac{\partial \varphi_{3}}{\partial q}\right) \\
+\varphi_{1}\left[\frac{\partial^{2} \eta_{u}}{\partial t^{2}}\left(\frac{\partial \varphi_{2}}{\partial q}\right)^{2}+\frac{\partial^{2} \eta_{u}}{\partial q^{2}}\left(\frac{\partial \varphi_{3}}{\partial q}\right)^{2}+2 \frac{\partial^{2} \eta_{u}}{\partial t \partial q} \frac{\partial \varphi_{2}}{\partial q} \frac{\partial \varphi_{3}}{\partial q}+\frac{\partial \eta_{u}}{\partial t} \frac{\partial^{2} \varphi_{2}}{\partial q^{2}}+\frac{\partial \eta_{u}}{\partial q} \frac{\partial^{2} \varphi_{3}}{\partial q^{2}}\right]
\end{gathered}
$$

and

$$
V(t, q) \eta_{u}^{V}=a(t) q^{2} \varphi_{1} \eta_{u}+b(t) q \varphi_{1} \eta_{u}+c(t) \varphi_{1} \eta_{u} .
$$

So, it's enough to have:

$$
\begin{gathered}
\theta^{2} \varphi_{1} \frac{\partial \varphi_{2}}{\partial t}-\theta^{2} \varphi_{1}\left(\frac{\partial \varphi_{3}}{\partial q}\right)^{2}+\frac{\theta^{4}}{2}\left(2 \frac{\partial \varphi_{1}}{\partial q} \frac{\partial \varphi_{2}}{\partial q}+\frac{\partial^{2} \varphi_{2}}{\partial q^{2}}\right)=0 \\
\frac{\theta^{4}}{2} \varphi_{1}\left(\frac{\partial \varphi_{2}}{\partial q}\right)^{2}=0 \\
\theta^{2} \varphi_{1} \frac{\partial \varphi_{3}}{\partial t}+\frac{\theta^{4}}{2}\left(2 \frac{\partial \varphi_{1}}{\partial q} \frac{\partial \varphi_{3}}{\partial q}+\varphi_{1} \frac{\partial^{2} \varphi_{3}}{\partial q^{2}}\right)=0 \\
\theta^{4} \frac{\partial \varphi_{2}}{\partial q} \frac{\partial \varphi_{3}}{\partial q}=0 \\
\theta^{2} \frac{\partial \varphi_{1}}{\partial t}+\frac{\theta^{4}}{2} \frac{\partial^{2} \varphi_{1}}{\partial q^{2}}-\varphi_{1}\left(a(t) q^{2}+b(t) q+c(t)\right)=0
\end{gathered}
$$


As $\varphi_{1}(0, q)=1$, the equation (1.3) gives us: $\left(\frac{\partial \varphi_{2}}{\partial q}\right)^{2}=0$, then

$$
\varphi_{2}=\varphi_{2}(t)
$$

and (1.5) is then automatically satisfied.

So, the equation (1.2) implies:

$\varphi_{1}(t, q)\left(\frac{\partial \varphi_{2}}{\partial t}-\left(\frac{\partial \varphi_{3}}{\partial q}\right)^{2}\right)=0$, then for all $(t, q)$ :

$$
\begin{aligned}
\frac{\partial \varphi_{2}}{\partial t} & =\left(\frac{\partial \varphi_{3}}{\partial q}\right)^{2} \\
\frac{\partial \varphi_{3}}{\partial q} & =A(t)
\end{aligned}
$$

$$
\varphi_{3}(t, q)=A(t) q+B(t)
$$

and

$$
\varphi_{2}(t)=\int_{0}^{t} A^{2}(s) d s
$$

then the equation (1.4) gives :

$$
\begin{aligned}
\varphi_{1} \frac{\partial \varphi_{3}}{\partial t}+\theta^{2} \frac{\partial \varphi_{1}}{\partial q} \frac{\partial \varphi_{3}}{\partial q} & =0 \\
\varphi_{1}(\dot{A}(t) q+\dot{B}(t))+\theta^{2} A(t) \frac{\partial \varphi_{1}}{\partial q} & =0 \\
\varphi_{1}(t, q)=k(t) e^{-\frac{1}{\theta^{2} A(t)}\left(\frac{\dot{A}(t)}{2} q^{2}+\dot{B}(t) q\right)} &
\end{aligned}
$$

therefore :

$$
\begin{gathered}
\frac{\partial \varphi_{1}}{\partial t}=\left\{\dot{k}(t)+\frac{k(t)}{\theta^{2} A(t)}\left(\frac{1}{2}\left(\frac{\dot{A}^{2}(t)}{A(t)}-\ddot{A}(t)\right) q^{2}+\left(\frac{\dot{A}(t)}{A(t)} \dot{B}(t)-\ddot{B}(t)\right) q\right)\right\} e^{-\frac{1}{\theta^{2} A(t)}\left(\frac{\dot{A}(t)}{2} q^{2}+\dot{B}(t) q\right),} \\
\frac{\partial \varphi_{1}}{\partial q}=\frac{-1}{\theta^{2}} \frac{\varphi_{1}}{A(t)}(\dot{A}(t) q+\dot{B}(t))
\end{gathered}
$$

and

$$
\frac{\partial^{2} \varphi_{1}}{\partial q^{2}}=\frac{1}{\theta^{4}} \frac{\varphi_{1}}{A^{2}(t)}\left(\dot{A}^{2}(t) q^{2}+2 \dot{A}(t) \dot{B}(t) q-\theta^{2} \dot{A}(t) A(t)+\dot{B}^{2}(t)\right),
$$

then the equation (1.6) becomes:

$$
\begin{aligned}
& k(t)\left(-\frac{1}{2} \frac{\ddot{A}(t)}{A(t)}+\frac{\dot{A}^{2}(t)}{A^{2}(t)}-a(t)\right) q^{2}+k(t)\left(-\frac{\ddot{B}(t)}{A(t)}+2 \dot{B}(t) \frac{\dot{A}(t)}{A^{2}(t)}-b(t)\right) q \\
& +\theta^{2} \dot{k}(t)-k(t) c(t)+\frac{k(t)}{2} \frac{\dot{B}^{2}(t)}{A^{2}(t)}-\theta^{2} \frac{k(t)}{2} \frac{\dot{A}(t)}{A(t)}=0 .
\end{aligned}
$$


As $k(t) \neq 0$, the equation (1.6) is equivalent to the following system :

$$
\left\{\begin{array}{l}
-\frac{1}{2} \frac{\ddot{A}(t)}{A(t)}+\frac{\dot{A}^{2}(t)}{A^{2}(t)}-a(t)=0 \\
-\frac{\ddot{B}(t)}{A(t)}+2 \dot{B}(t) \frac{\dot{A}(t)}{A^{2}(t)}-b(t)=0 \\
\theta^{2} \dot{k}(t)-k(t) c(t)+\frac{k(t)}{2} \frac{\dot{B}^{2}(t)}{A^{2}(t)}-\theta^{2} \frac{k(t)}{2} \frac{\dot{A}(t)}{A(t)}=0
\end{array}\right.
$$

knowing that :

$$
\left\{\begin{array}{l}
A(0)=1 \\
B(0)=0 \\
k(0)=1 \\
\dot{A}(0)=0 \\
\dot{B}(0)=0
\end{array}\right.
$$

This is due to initial conditions at time $t$ on the functions $\varphi_{1}(t, q), \varphi_{2}(t, q)$, and $\varphi_{3}(t, q)$.

Let

$$
A(t)=e^{\int_{0}^{t} \rho(s) d s}
$$

then

$$
\rho(t)=\frac{\dot{A}(t)}{A(t)}
$$

and

$$
\dot{\rho}(t)+\rho^{2}(t)=\frac{\ddot{A}(t)}{A(t)},
$$

therefore the first equation of (1.10) is equivalent to a Riccati equation :

$$
\left\{\begin{array}{l}
\rho^{2}(t)-\dot{\rho}(t)=2 a(t) \\
\rho(0)=\frac{\dot{A}(0)}{A(0)}=0 .
\end{array}\right.
$$

The second equation of (1.10) gives: $2 \frac{\dot{A}(t)}{A(t)} \dot{B}(t)-\ddot{B}(t)=A(t) b(t)$,

then

$$
\begin{aligned}
\left(A^{-2}(t) \dot{B}(t)\right)^{\prime} & =-2 A^{-3}(t) \dot{A}(t) \dot{B}(t)+A^{-2}(t) \ddot{B}(t) \\
& =-A^{-2}(t)\left(2 \frac{\dot{A}(t)}{A(t)} \dot{B}(t)-\ddot{B}(t)\right) \\
& =-A^{-2}(t)(A(t) b(t)) \\
& =-\frac{b(t)}{A(t)},
\end{aligned}
$$

therefore $B(t)$ is the solution of :

$$
\left\{\begin{array}{l}
\dot{B}(t)=-A^{2}(t) \int_{0}^{t} \frac{b(s)}{A(s)} d s \\
B(0)=0 .
\end{array}\right.
$$


Let $\Delta(t)=\int_{0}^{t} \frac{b(s)}{A(s)} d s$ and define $k$ by:

$$
\left\{\begin{aligned}
\dot{k}(t) & =\frac{k(t)}{\theta^{2}}\left(c(t)-\frac{1}{2} A^{2}(t) \Delta^{2}(t)+\frac{\theta^{2}}{2} \rho(t)\right) \\
k(0) & =1 .
\end{aligned}\right.
$$

this makes the first equation of (1.7) satisfied.

Remark 1.2. If $a(t), b(t)$ and $c(t)$ were constants, then we could apply the method of Rosencrans (cf. [6]).

\subsection{Particular cases.}

i) $\mathbf{V}(\mathbf{t}, \mathbf{q})=\mathbf{a}(\mathbf{t}) \mathbf{q}^{2}($ semiclassical case $)$.

Then

$$
\begin{gathered}
B(t)=0, \Delta(t)=0, \\
k(t)=k(0) e^{\frac{1}{\theta^{2}} \int_{0}^{t}\left(c(s)-\frac{1}{2} A^{2}(s) \Delta^{2}(s)+\frac{\theta^{2}}{2} \rho(s)\right) d s} \\
=e^{\frac{1}{2} \int_{0}^{t} \frac{\dot{A}(s)}{A(s)}} d s \\
=\sqrt{A(t)}
\end{gathered}
$$

and

$$
\eta_{u}^{V}(t, q)=\sqrt{A(t)} e^{-\frac{q^{2}}{2 \theta^{2}} \rho(t)} \eta_{u}\left(\varphi_{2}(t), A(t) q\right)
$$

is the solution of $\left(C_{1}^{(V)}\right)$ such that $\eta_{u}^{V}(0, q)=\eta_{u}(0, q)=u(q)$.

In this case, as in the next one, the formulas really involve only $A(t)$.

$\mathbf{i}-\mathbf{a}) \mathbf{V}(\mathbf{t}, \mathbf{q})=\frac{\omega^{2}}{2} \mathbf{q}^{2}$.

Then

$$
\rho(t)=-\omega \tanh (\omega t), A(t)=\frac{1}{\cosh (\omega t)}, \varphi_{2}(t, q)=\frac{1}{\omega} \tanh (\omega t)
$$

and according to i)

$$
\eta_{u}^{V}(t, q)=\frac{1}{\sqrt{\cosh (\omega t)}} e^{\frac{\omega}{2 \theta^{2}} \tanh (\omega t) q^{2}} \eta_{u}\left(\frac{1}{\omega} \tanh (\omega t), \frac{q}{\cosh (\omega t)}\right)
$$

is the solution of $\left(C_{1}^{(V)}\right)$.

This result is due to Zambrini (cf.[7], p.227, (51)).

The existence of such a formula was mentioned in [5], p. 96, and the similar result for the Schrödinger equation with quadratic potential (with $\cos (\omega t)$ taking the place of $\cosh (\omega t))$ appeared in [5], p. 83.

ii) $\mathbf{V}(\mathbf{t}, \mathbf{q})=\lambda(\mathbf{t}) \mathbf{q}$.

Then $\rho(t)=0, A(t)=1, \dot{B}(t)=-\int_{0}^{t} \lambda(s) d s, B(t)=\int_{0}^{t} \dot{B}(u) d u=$ $-\int_{0}^{t}\left(\int_{0}^{u} \lambda(s) d s\right) d u$

and

$$
\eta_{u}^{V}(t, q)=k(t) e^{-\frac{1}{\theta^{2}} \dot{B}(t) q} \eta_{u}(t, q+B(t))
$$

is the solution of $\left(C_{1}^{(V)}\right)$, such that $\eta_{u}^{V}(0, q)=\eta_{u}(0, q)=u(q)$. 
ii $-\mathbf{a}) \mathbf{V}(\mathbf{t}, \mathbf{q})=\lambda \mathbf{q}$

Then $A(t)=1, \Delta(t)=\lambda t, \dot{B}(t)=-\lambda t, B(t)=-\frac{\lambda}{2} t^{2}, k(t)=e^{-\frac{\lambda^{2}}{6 \theta^{2}} t^{3}}$ and

$$
\eta_{u}^{V}(t, q)=e^{-\frac{1}{\theta^{2}}\left(\frac{\lambda^{2}}{6} t^{3}-\lambda t q\right)} \eta_{u}\left(t, q-\frac{\lambda t^{2}}{2}\right)
$$

is the solution of $\left(C_{1}^{(V)}\right)$.

We hereby recover a result of Lescot and Zambrini (cf.[4], p.219, (1)).

\section{ISOVECTORS}

Let $\mathcal{G}_{V}$ defined by :

$$
\mathcal{G}_{V}=\left\{N \mid \mathcal{L}_{N}(I) \subset I\right\}
$$

and $\mathcal{H}_{V}$ by :

$$
\mathcal{H}_{V}=\left\{N \in \mathcal{G}_{V} \mid \frac{\partial N^{S}}{\partial S}=0\right\} .
$$

These definitions are taken from Lescot-Zambrini (cf.[4]).

We remind the reader that a $\mathcal{G}_{V}$ is a Lie algebra for the usual Lie bracket of vectors fields, and $\mathcal{H}_{V}$ is a Lie subalgebra of $\mathcal{G}_{V}$ (cf.[4], p.210-211).

According to Lescot-Zambrini (cf.[4], p.214-215, 3.9'- 3.29), for all $N \in \mathcal{H}_{V}$, we can write :

$$
\begin{gathered}
N^{q}:=\frac{1}{2} \dot{T}_{N}(t) q+\ell_{N}(t) \\
N^{t}:=T_{N}(t) \\
N^{S}:=h(q, t, S),
\end{gathered}
$$

for $\sigma(t), l(t)$ and $T_{N}(t)$ satisfying :

$(2.1)-\frac{1}{4} \dddot{T}_{N} q^{2}-\ddot{\ell} q+\dot{\sigma}+T_{N} \frac{\partial V}{\partial t}+\frac{1}{2} \dot{T}_{N} q \frac{\partial V}{\partial q}+\ell \frac{\partial V}{\partial q}+\dot{T}_{N} V-\frac{\theta^{2}}{4} \ddot{T}_{N}=0$.

Let us set $l_{N}=l$.

Proposition 2.1. Let $\mathcal{K}_{V}$ defined by :

$$
\mathcal{K}_{V}=\left\{N \in \mathcal{H}_{V} / \ell_{N}=0\right\},
$$

then $\mathcal{K}_{V}$ is a Lie subalgebra of $\mathcal{H}_{V}$.

Proof. Let $N_{1}, N_{2} \in \mathcal{K}_{V}$;one has 


$$
\begin{aligned}
{\left[N_{1}, N_{2}\right]^{q} } & =\left[N_{1}, N_{2}\right](q) \\
& =N_{1}\left(N_{2}^{q}\right)-N_{2}\left(N_{1}^{q}\right) \\
& =N_{1}^{q} \frac{\partial N_{2}^{q}}{\partial q}+N_{1}^{t} \frac{\partial N_{2}^{q}}{\partial t}-\left(N_{2}^{q} \frac{\partial N_{1}^{q}}{\partial q}+N_{2}^{t} \frac{\partial N_{1}^{q}}{\partial t}\right) \\
& =\frac{1}{2} \dot{T}_{N_{2}}\left(\frac{1}{2} \dot{T}_{N_{1}} q+\ell_{N_{1}}(t)\right)+T_{N_{1}}\left(\frac{1}{2} \ddot{T}_{N_{2}} q+\dot{\ell}_{N_{2}}\right)-\frac{1}{2} \dot{T}_{N_{1}}\left(\frac{1}{2} \dot{T}_{N_{2}} q+\ell_{N_{2}}(t)\right) \\
& -T_{N_{2}}(t)\left(\frac{1}{2} \ddot{T}_{N_{1}} q+\dot{\ell}_{N_{1}}\right) \\
& =\left(T_{N_{1}}(t) \dot{\ell}_{N_{2}}-T_{N_{2}}(t) \dot{\ell}_{N_{1}}+\frac{1}{2}\left(\ell_{N_{1}}(t) \dot{T}_{N_{2}}-\ell_{N_{2}}(t) \dot{T}_{N_{1}}\right)\right) \\
& +\frac{1}{2}\left(T_{N_{1}}(t) \ddot{T}_{N_{2}}(t)-T_{N_{2}}(t) \ddot{T}_{N_{1}}(t)\right) q
\end{aligned}
$$

and $\ell_{\left[N_{1}, N_{2}\right]}=T_{N_{1}}(t) \dot{\ell}_{N_{2}}-T_{N_{2}}(t) \dot{\ell}_{N_{1}}+\frac{1}{2}\left(\ell_{N_{1}}(t) \dot{T}_{N_{2}}-\ell_{N_{2}}(t) \dot{T}_{N_{1}}\right)=0$.

Then $\mathcal{K}_{V}$ is stable by the Lie bracket, therefore $\mathcal{K}_{V}$ is a Lie subalgebra of $\mathcal{H}_{V}$.

Lemma 2.2. If $V_{1}(t, q)=V_{2}(t, q)+\frac{C}{q^{2}}($ for $C$ a constant $)$, then $\mathcal{K}_{V_{1}}=\mathcal{K}_{V_{2}}$.

Proof. Replacing $V_{1}(t, q)$ by its value in equation (2.1), we get:

$$
\begin{aligned}
& -\frac{1}{4} \dddot{T}_{N} q^{2}-\ddot{\ell} q+\dot{\sigma}+T_{N} \frac{\partial V_{2}}{\partial t}+\frac{1}{2} \dot{T}_{N} q\left(\frac{\partial V_{2}}{\partial q}-2 \frac{C}{q^{3}}\right)+\ell\left(\frac{\partial V_{2}}{\partial q}-2 \frac{C}{q^{3}}\right)+\dot{T}_{N}\left(V_{2}+\frac{C}{q^{2}}\right) \\
\Leftrightarrow & -\frac{\theta^{2}}{4} \ddot{T}_{N}=0 \\
& -\frac{1}{4} \dddot{T}_{N} q^{2}-\ddot{\ell} q+\dot{\sigma}+T_{N} \frac{\partial V_{2}}{\partial t}+\frac{1}{2} \dot{T}_{N} q \frac{\partial V_{2}}{\partial q}+\ell \frac{\partial V_{2}}{\partial q}-\frac{2 l C}{q^{3}}+\dot{T}_{N} V_{2}-\frac{\theta^{2}}{4} \ddot{T}_{N}=0 .
\end{aligned}
$$

Therefore, if $N \in \mathcal{K}_{V_{2}}$, as $l_{N}=0$, we see that $n$ satisfies (2.1) for $V=V_{1}$. Then $V_{1}$ et $V_{2}$ give the same isovectors such as $\ell_{N}=0$.

Therefore :

$$
\mathcal{K}_{V_{1}}=\mathcal{K}_{V_{2}}
$$

Corollary 2.3. If $V_{1}=\frac{C}{q^{2}}(C \neq 0)$ and $V_{2}=0$, then $\mathcal{H}_{V_{1}}=\mathcal{K}_{V_{1}}=\mathcal{K}_{V_{2}}$ and therefore: $\mathcal{H}_{V_{1}} \subset \mathcal{H}_{V_{2}}$.

Proof. Let $N \in \mathcal{H}_{V_{1}}$, the equation (2.1) becomes:

$$
-\frac{1}{4} \dddot{T}_{N} q^{2}-\ddot{\ell} q+\dot{\sigma}+\frac{1}{2} \dot{T}_{N} q\left(-2 \frac{C}{q^{3}}\right)+\ell\left(-2 \frac{C}{q^{3}}\right)+\dot{T}_{N}\left(\frac{C}{q^{2}}\right)-\frac{\theta^{2}}{4} \ddot{T}_{N}=0,
$$

that is

$$
-\frac{1}{4} \dddot{T}_{N} q^{2}-\ddot{\ell} q+\dot{\sigma}-\frac{\theta^{2}}{4} \ddot{T}_{N}-2 \frac{\ell C}{q^{3}}=0 .
$$

As $T_{N}, \ell$ and $\sigma$ depend only on $t$, the system is equivalent to: 


$$
\left\{\begin{array}{r}
2 C \ell=0 \\
\ddot{\ell}=0 \\
\dot{\sigma}=\frac{\theta^{2}}{4} \ddot{T}_{N} \\
\dddot{T}_{N}=0 .
\end{array}\right.
$$

But $C \neq 0$, therefore $\ell_{N}=0$ and

$$
\mathcal{H}_{V_{1}}=\mathcal{K}_{V_{1}} \underset{\text { (Lemma 2.2) }}{=} \mathcal{K}_{V_{2}} \subset \mathcal{H}_{V_{2}}
$$

Remark 2.4. Thus, we recover another result of Lescot and Zambrini(cf.[4], p.220, $(3))$.

\section{REFERENCES}

1. M. Flato, J. Simon, H. Snellmann and D. Sternheimer, Simple facts about analytic vectors and integrability, Annales Scientifiques de l'E.N.S. $4^{e}$ srie, tome 5, $n^{o} 3$ (1972), p. 423-434.

2. P.Lescot, H. Quintard and J.-C. Zambrini , Solving stochastic differential equations with Cartan's exterior differential system (preprint, 2015).

3. P.Lescot and J.-C.Zambrini, Isovectors for the Hamilton-Jacobi-Bellman Equation, Formal Stochastic Differentials and First Integrals in Euclidean Quantum Mechanics, Proceedings of the Ascona conference (2002), 187-202. Birkhaüser (Progress in Probability, vol 58), 2004.

4. P.Lescot and J.-C. Zambrini, Probabilistic deformation of contact geometry, diffusion processes and their quadratures, Seminar on Stochastic Analysis, Random Fields and applications V, 203-226, Birkhaüser(Progress in Probability, vol. 59), 2008.

5. W. Miller, Jr., Symmetry and separation of variables, Encyclopedia of Mathematics and its Applications 4, Cambridge University Press (2012).

6. S.I.Rosencrans, Perturbation Algebra of an Elleptic Operator, Journal of Mathematical Analysis and Applications 56, 317-329 (1976).

7. J.-C.Zambrini, From the geometry of parabolic PDE to the geometry of SDE, Seminar on Mathematical analysis of random phenomena, 213-230, World Sci. Publ. Hackensack, NJ, 2007.

Laboratoire de Mathématiques Raphal Salem, UMR 6085 CNRS-Université de Rouen, Avenue de l’Université, BP.12, Technopôle du Madrillet, F76801 Saint-Étienne-duRouvray, E-mail: Mhamed.machHour@gmail.com \& PaUl.Lescot@Univ-Rouen.Fr, 